\title{
The effects of experimental uncertainty in parameterizing air-sea gas exchange using tracer experiment data
}

\author{
W. E. Asher \\ University of Washington, Seattle, Washington, USA \\ Received: 4 July 2008 - Published in Atmos. Chem. Phys. Discuss.: 3 September 2008 \\ Revised: 6 November 2008 - Accepted: 17 November 2008 - Published: 9 January 2009
}

\begin{abstract}
It is not practical to measure air-sea gas fluxes in the open ocean for all conditions and areas of interest. Therefore, in many cases fluxes are estimated from measurements of air-phase and water-phase gas concentrations, a measured environmental forcing function such as wind speed, and a parameterization of the air-sea transfer velocity in terms of the environmental forcing function. One problem with this approach is that when direct measurements of the transfer velocity are plotted versus the most commonly used forcing function, wind speed, there is considerable scatter, leading to a relatively large uncertainty in the flux. Because it is known that multiple processes can affect gas transfer, it is commonly assumed that this scatter is caused by single-forcing function parameterizations being incomplete in a physical sense. However, scatter in the experimental data can also result from experimental uncertainty (i.e., measurement error). Here, results from field and laboratory results are used to estimate how experimental uncertainty contributes to the observed scatter in the measured fluxes and transfer velocities as a function of environmental forcing. The results show that experimental uncertainty could explain half of the observed scatter in field and laboratory measurements of air-sea gas transfer velocity.
\end{abstract}

\section{Introduction}

Advances in techniques for measuring air-sea fluxes have resulted in several new oceanic data sets of oceanic gas fluxes (Edson et al., 2004; Ho et al., 2006; Jacobs et al., 1999; McGillis et al., 2001a, b; Nightingale et al., 2000a, b; Wanninkhof et al., 1993, 1997, 2004). In addition, novel experimental methodologies and detailed microphysical pro-

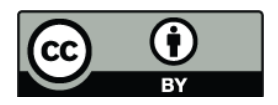

Correspondence to: W. E. Asher (asher@apl.washington.edu) cess studies have provided new information concerning the fundamental mechanisms controlling air-water gas exchange (Asher and Litchendorf, 2009; Herlina and Jirka, 2004, 2008; McKenna and McGillis, 2004; Münsterer and Jähne, 1998; Siddiqui et al., 2004; Woodrow and Duke, 2001; Zappa et al., 2004). These field and laboratory data have been used in developing and testing air-sea gas exchange dependencies and have allowed commonly used conceptual models to be tested. However, even with the advances in the understanding of the process and the additional experimental capabilities, variability in both laboratory and field data as a function of a particular variable characterizing the major forcing functions (e.g., wind stress) has made development of a robust method for parameterizing the gas transfer velocity difficult.

In general, the air-sea flux of a sparingly soluble nonreactive gas at low to moderate wind speeds can be written as the product of a kinetic term, the air-sea gas transfer velocity $k_{L}\left(\mu \mathrm{m} \mathrm{s}^{-1}\right)$, and a thermodynamic driving force defined in terms of the disequilibrium in chemical potential of the gas between the ocean and the atmosphere. This driving force is commonly expressed in terms of the air-water partial pressure difference, $\Delta P(\mathrm{kPa})$ assuming that most gases of interest will behave ideally so that the fugacity in each phase is equal to their partial pressure in that phase. Although there is some evidence that errors in $\Delta P$ can affect the measurment of $k_{L}$ (Jacobs et al., 2002), this is by no means conclusive and this discussion will focus on the kinetic term $k_{L}$.

It is well understood that in the absence of bubbles $k_{L}$ depends on both the molecular diffusivity, $D\left(\mathrm{~m}^{2} \mathrm{~s}^{-1}\right)$, of the gas in the aqueous phase and on the water-side turbulence very close to the free surface (Davies, 1972). However, the details of these dependencies as they relate to the particulars of gas transfer at the ocean surface are not well known and still subject to considerable debate. For example, it is clear that the presence of naturally occurring surface active material (which will be referred to here as "surfactants" for short) can inhibit air-water gas transfer (Frew et al., 1990).

Published by Copernicus Publications on behalf of the European Geosciences Union. 


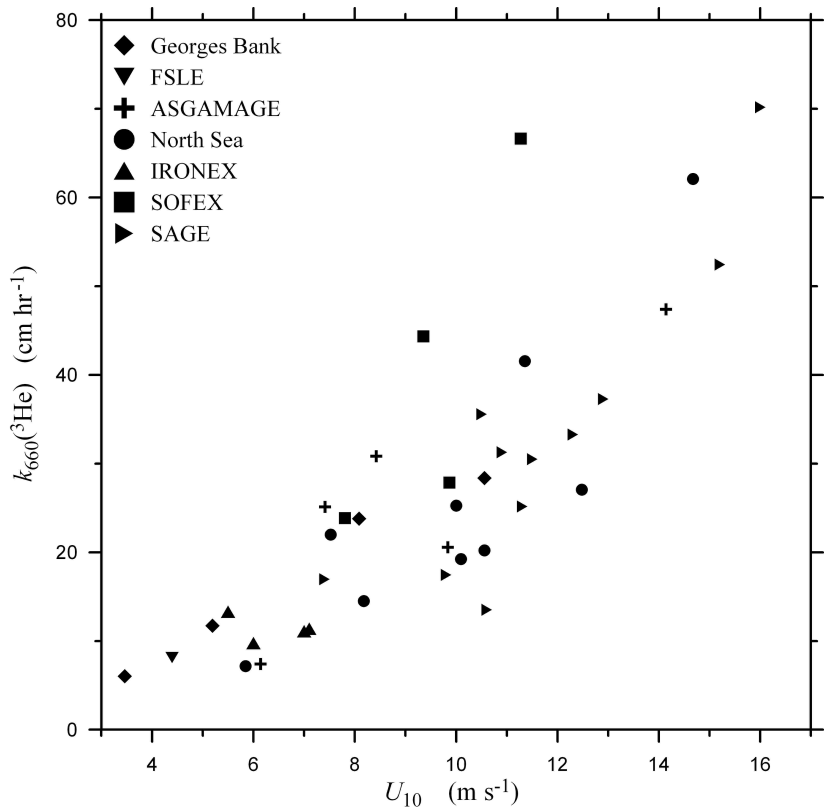

Fig. 1. Available oceanic measurements of the air-sea gas transfer velocity made using the purposeful dual-tracer method normalized to a common Schmidt number of $660, k_{660}\left({ }^{3} \mathrm{He}\right)$, plotted as a function of wind speed. The data key is shown on the figure. In the key: Georges Bank is data from Wanninkhof et al. (1993); FSLE is data from Wanninkhof et al. (1997); ASGAMAGE is data from Jacobs et al. (1999); North Sea is data from Nightingale et al. (2000b), IRONEX is data from Nightingale et al. (2000a); SOFEX is data from Wanninkhof et al. (2004); and SAGE is data from Ho et al. (2006).

However, it is less clear that field measurements of $k_{L}$ can be easily partitioned into those made under surfactant-impacted conditions and those made under so-called clean conditions. Even more uncertain is how to best parameterize the role of turbulence in influencing the magnitude of $k_{L}$.

Under most conditions, the wind stress plays a dominant role in providing the turbulence kinetic energy involved in promoting gas exchange. Therefore, wind speed, $U$, has long been used to parameterize $k_{L}$. Figure 1 shows $k_{L}$ measured in the ocean using the purposeful dual-tracer method (Watson et al., 1991) plotted as a function of $U$. (The data in the figure were compiled from Wanninkhof et al. (1993, 1997, 2004), Jacobs et al. (1999), Nightingale et al. (2000a, b) and Ho et al. (2006) and have all been scaled to a common diffusivity equal to carbon dioxide in seawater at $293.15 \mathrm{~K}$, defined here in terms of the Schmidt number (660), assuming that $k_{L}$ is proportional to $D^{1 / 2}$ ). When the values for the scaled transfer velocity, $k_{660}$, at a particular $U$ are compared, the data are considerably scattered. It can be argued that the overall dependence follows either a polynomial dependence or a segmented linear dependence with $U$. Unfortunately, there is too much scatter in the data to allow the data to provide a definitive selection between any of the available gas exchange parameterizations.
Figure 1 typifies the problem in attempting to develop a method for accurately estimating $k_{L}$ from an easily measured environmental parameter. The data in the figure represent measurements of the highest quality, collected by meticulous and careful groups. Because of this, it is assumed that much of the scatter in the data represents variability in the transfer velocity imposed by variability in the environmental conditions. This could occur if, for example, the levels of aqueous-phase turbulence generated at a particular value of $U$ depend on factors other than the $U$ itself. This explanation seems logical on an intuitive level, but the fundamental measurements of near-surface oceanic turbulence necessary to support it are not available. So it could be that the scatter in Fig. 1 could simply represent experimental uncertainty rather than environmental variability.

The purpose of this paper is to explore whether it is possible to explain the scatter in various measurements of the $k_{L}$ simply in terms of the uncertainty in measuring the underlying parameters. This will be done using the dual-tracer data shown in Fig. 1 and using gas transfer data collected in a wind-wave tunnel.

\section{Theory}

In the case where a non-reactive gaseous tracer is injected into a known volume of water, the change in concentration with respect to time due to the water-to-air gas flux can be written as

$\frac{d C_{B}}{d t}=\frac{k_{L} A}{V}\left(K_{H} P_{A}-C_{B}\right)$

where $C_{B}$ is the bulk-phase concentration of the tracer gas $\left(\mathrm{mol} \mathrm{m}^{-3}\right), A$ is the surface area of the water through which gas exchange occurs $\left(\mathrm{m}^{2}\right), V$ is the total water volume $\left(\mathrm{m}^{3}\right), K_{H}$ is the aqueous-phase solubility of the gas (mol m${ }^{-3} \mathrm{kPa}^{-1}$ ), and $P_{A}$ is the partial pressure of the gas in the air phase $(\mathrm{kPa})$. The product $K_{H} P_{A}$ defines the equilibrium saturation concentration of the gas, $C_{S}$. Integration of Eq. (1) shows that $k_{L}$ can be written as

$k_{L}=\frac{V}{A \Delta t} \ln \left(\frac{C_{S}-C_{0}}{C_{S}-C_{B}}\right)$

where $\Delta t$ is the time difference and $C_{0}$ is the concentration of the tracer gas at time $t=0$. From Eq. (2), a plot of the quantity $-\ln \left(\left(C_{S}-C_{0}\right) /\left(C_{S}-C_{B}\right)\right.$ versus time will result in a straight line with slope equal to $k_{L} A / V$.

A similar relation exists for the analysis of purposeful dual-tracer method (PDTM) data collected during oceanic air-sea gas exchange measurements. In these experiments, the two volatile tracer gases sulfur hexafluoride $\left(\mathrm{SF}_{6}\right)$ and helium-3 $\left({ }^{3} \mathrm{He}\right)$ are injected into the surface mixed layer. Their concentrations are then measured as a function of time and the transfer velocity of ${ }^{3} \mathrm{He}, k_{L}\left({ }^{3} \mathrm{He}\right)$, can be estimated from the change in the concentration ratio of the two tracers. 


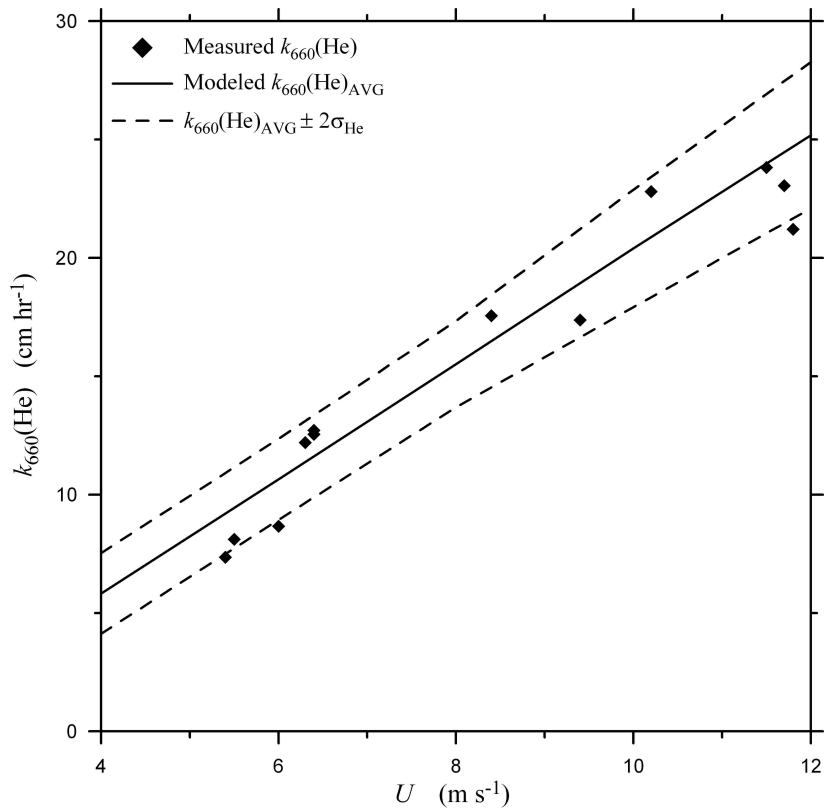

Fig. 2. The air-water gas transfer velocity for He normalized to $\mathrm{Sc}=600, k_{660}(\mathrm{He})$, measured during FEDS plotted as a function of wind speed. Also shown are values for $k_{660}(\mathrm{He})_{\mathrm{AVG}}$ produced by the Monte Carlo model described in Sect. 3. The data key is shown on the figure. The dashed lines represent the values bounding $90 \%$ of the $k_{660}(\mathrm{He})_{\mathrm{AVG}}$ values produced by the Monte Carlo model.

If the atmospheric concentration of both gases is assumed to be zero (Wanninkhof et al., 1993), this relation has the form

$k_{L}\left({ }^{3} \mathrm{He}\right)=-\frac{h}{\Delta t} \Delta\left\{\ln \left(\frac{\left[{ }^{3} \mathrm{He}\right]}{\left[\mathrm{SF}_{6}\right]}\right)\right\}\left\{1-\left[\frac{S c\left({ }^{3} \mathrm{He}\right)}{\mathrm{Sc}\left(\mathrm{SF}_{6}\right)}\right]^{1 / 2}\right\}^{-1}$

where $h$ is the mixed layer depth, $\Delta t$ is the time interval over which the change in concentrations are measured, $\left[{ }^{3} \mathrm{He}\right]$ and $\left[\mathrm{SF}_{6}\right]$ are the concentrations of ${ }^{3} \mathrm{He}$ and $\mathrm{SF}_{6}$, respectively, and $\mathrm{Sc}\left({ }^{3} \mathrm{He}\right)$ and $\mathrm{Sc}\left(\mathrm{SF}_{6}\right)$ are the Schmidt numbers of ${ }^{3} \mathrm{He}$ and $\mathrm{SF}_{6}$, respectively. In specific, the term $\Delta\left\{\ln \left(\left[{ }^{3} \mathrm{He}\right] /\left[\mathrm{SF}_{6}\right]\right)\right\}$ in Eq. (3) should be interpreted as $\ln \left(\left[{ }^{3} \mathrm{He}\right]_{t_{2}} /\left[\mathrm{SF}_{6}\right]_{t_{2}}\right)-\ln \left(\left[{ }^{3} \mathrm{He}\right]_{t_{1}} /\left[\mathrm{SF}_{6}\right]_{t_{1}}\right)$ where the subscripts $t_{1}$ and $t_{2}$ imply the concentrations measured at times $t_{1}$ and $t_{2}$, respectively. The "dual-tracer term" defined by 1 $\left[\mathrm{Sc}(3 \mathrm{He}) / \mathrm{Sc}\left(\mathrm{SF}_{6}\right)\right]^{1 / 2}$ is 0.64 at $278 \mathrm{~K}$ for seawater and 0.61 at $298 \mathrm{~K}$ using $\mathrm{Sc}\left({ }^{3} \mathrm{He}\right)$ calculated as outlined in Jähne et al. (1987) and $\mathrm{Sc}\left(\mathrm{SF}_{6}\right)$ from King and Saltzman (1995)

Applying either Eq. (2) or (3) is straightforward and the theoretical basis for each are not in dispute (with possible exception being the correct value of the exponent for the Schmidt number term). However, because of the logarithmic relationship involving the concentrations and the fact that the change in concentration with respect to time can be relatively small, both equations are sensitive to measurement uncertainty.

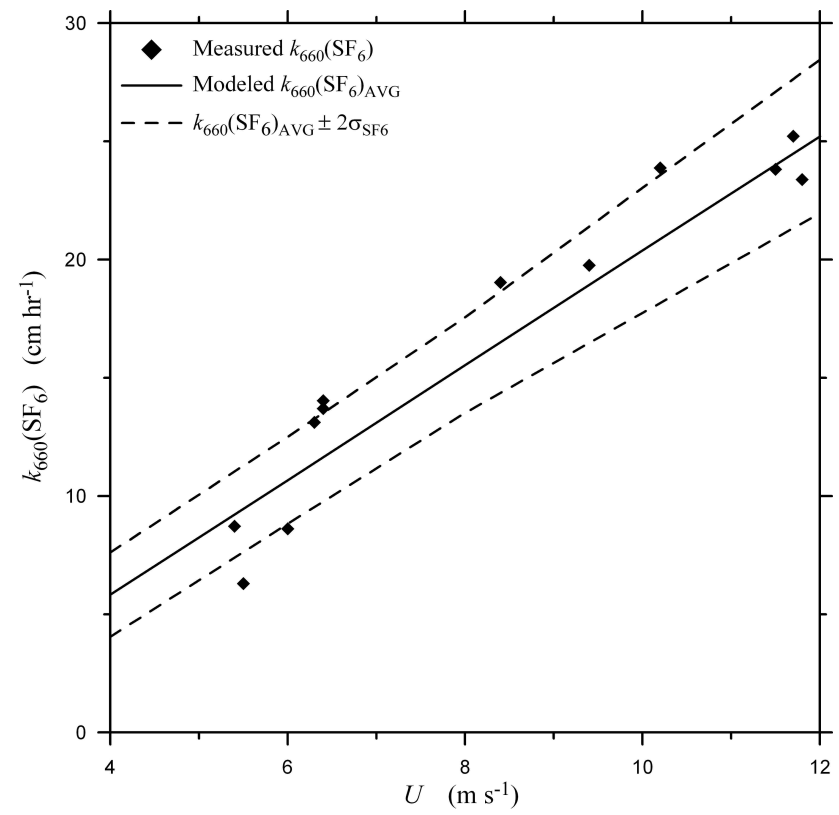

Fig. 3. The air-water gas transfer velocity for $\mathrm{SF}_{6}$ normalized to $\mathrm{Sc}=660, k_{660}\left(\mathrm{SF}_{6}\right)$, measured during FEDS plotted as a function of wind speed. Also shown are values for $k_{660}\left(\mathrm{SF}_{6}\right)_{\text {AVG }}$ produced by the Monte Carlo model described in Sect. 3. The data key is shown on the figure. The dashed lines represent the values bounding $90 \%$ of the $k_{660}\left(\mathrm{SF}_{6}\right)_{\mathrm{AVG}}$ values produced by the Monte Carlo model.

In laboratory experiments, it is common to simultaneously measure $k_{L}$ for several gases. From these data, it is possible to estimate the dependence of $k_{L}$ on molecular diffusivity. Conceptual models for air-water gas transfer assume that this dependence can be written as

$k_{L}=a\left(\frac{v}{D}\right)^{-n} f(Q, L)$

where $a$ and $n$ are constants, $v$ is the kinematic viscosity of water, and $f(Q, L)$ symbolizes the as yet unspecified dependence of $k_{L}$ on the turbulence velocity and length scales. The ratio $v / D$ is the Schmidt number, $\mathrm{Sc}$, and it will be used from this point in place of $D$. Depending on the conceptual model, $n$ can range from $1 / 2$ to $2 / 3$ with the lower value usually associated with gas exchange through a clean water surface and higher values associated with transfer through surfactant influenced surfaces. Therefore, the dependence of $k_{L}$ on Sc provides very useful information on the transfer process and it is highly desirable to be able to estimate $n$ from experimental data.

Using Eq. (4), it can be shown that if the $k_{L}$ values for two gases with different Sc numbers are known, $n$ is equal to

$n=\frac{\ln \left[k_{L}(1) / k_{L}(2)\right]}{\ln [\operatorname{Sc}(2) / \operatorname{Sc}(1)]}$ 
where the (1) and (2) refer to the parameter for the two respective gases. As is the case for calculating $k_{L}$ itself, Eq. (5) is also sensitive to measurement errors because Sc for most gases does not vary by a large amount.

\section{Wind-wave tunnel measurements}

The Flux Exchange Dynamics Study (FEDS) was conducted in 1998 at the Air-Sea Interaction Research Facility (ASIRF) wind-wave tunnel at the US National Aeronautics and Space Agency, Goddard Space Flight Center, Wallops Flight Facility in Wallops Island, Virginia. These measurements have been described in detail elsewhere (Zappa et al., 2004) and only a brief description will be provided here. During the study, $k_{L}$ was measured for $\mathrm{SF}_{6}$ and helium (He) using gas chromatography to determine aqueous-phase gas concentrations. Method precision for measuring gas concentrations was found to be $\pm 3 \%$ for $\mathrm{SF}_{6}$ and $\pm 7 \%$ for He. Figure 2 shows transfer velocities for He normalized to $\mathrm{Sc}=660$ assuming $n=1 / 2, k_{660}(\mathrm{He})$, measured during FEDS plotted as a function of wind speed, $U$. Figure 3 shows transfer velocities measured during FEDS for $\mathrm{SF}_{6}$ normalized to $\mathrm{Sc}=660$, $k_{660}\left(\mathrm{SF}_{6}\right)$, also plotted versus $U$. Sc values for $\mathrm{SF}_{6}$ were taken from King and Saltzman (1995) and Sc for He was taken from Wanninkhof (1992). Uncertainty in $\mathrm{Sc}(\mathrm{He})$ is $2.1 \%$ (Jähne et al., 1987) and $4.2 \%$ for $\mathrm{Sc}\left(\mathrm{SF}_{6}\right)$ (King and Saltzman, 1995), although uncertainties in Sc were neglected here since they will not affect the variability in the resulting transfer velocities.

As was seen in the field data in Fig. 1, there is some scatter in the measured values of the transfer velocity when plotted versus $U$. However, in contrast to the field data, $k_{L}$ in the wind-wave tunnel increases approximately linearly with $U$. What is not clear from the data is whether the scatter in $k_{L}$ reflect experimental noise or day-to-day variability in the conditions affecting gas transfer in the wind tunnel. In order to understand the relative roles experimental uncertainty and environmental variability play in governing the scatter in measured $k_{L}$ values, a Monte Carlo-type simulation was performed using the known experimental uncertainties to estimate the variability in $k_{L}$ that would be expected given the uncertainties in the gas concentrations.

The first step in this procedure was to use the experimental data in Figs. 2 and 3 to produce a linear relation for estimating $k_{L}$ from wind speed in the ASIRF wind-wave tunnel. Least-squares linear regression of the combined gas transfer data set showed that $k_{L}$ at a particular value of $\mathrm{Sc}, k_{L}(\mathrm{Sc})$, could be calculated as

$k_{L}(\mathrm{Sc})=\left(\frac{660}{\mathrm{Sc}}\right)^{1 / 2}(6.73 U-10.76)$ for $k_{L}(\mathrm{Sc})$ in $\mu \mathrm{m} \mathrm{s}^{-1}$ and $U$ in $\mathrm{m} \mathrm{s}^{-1}$ with the coefficient of determination for the regression being 0.91 and a standard error of the fit equal to $5.29 \mu \mathrm{m} \mathrm{s}^{-1}$. Then, Eq. (2) was rewritten in the form

$C_{B}=C_{B}-\left(C_{S}-C_{0}\right) \exp \left(-\frac{A k_{L}(\mathrm{Sc}) t}{V}\right)$

so that gas concentrations for a gas with a particular Schmidt number could be predicted as a function of $t$. Concentrations for $\mathrm{He}$ and $\mathrm{SF}_{6}$ were predicted at five times with time steps on the order of $2000 \mathrm{~s}$ so that the number of modeled concentrations and their time steps were equal to the experimental sampling rate used in the actual experiments. (It should be noted that simulating experiments with extremely long time steps where gas concentrations decrease to within $10 \%$ of their equilibrium values greatly decreases the effect of measurement error.) Then, the predicted concentrations were modified by adding or subtracting a random amount determined by the measurement error for that particular gas.

For the Monte Carlo simulations, 1000 separate sets of concentrations with independent experimental uncertainties were produced for each gas at $U=4 \mathrm{~m} \mathrm{~s}^{-1}, 6 \mathrm{~m} \mathrm{~s}^{-1}$, $8 \mathrm{~m} \mathrm{~s}^{-1}, 10 \mathrm{~m} \mathrm{~s}^{-1}$, and $12 \mathrm{~m} \mathrm{~s}^{-1}$. These concentrations were then used in Eq. (2) to calculate $k_{660}\left(\mathrm{SF}_{6}\right)$ and $k_{660}(\mathrm{He})$. The mean value of the model-calculated transfer velocities, $k_{660}(\mathrm{He})_{\mathrm{AVG}}$ and $k_{660}\left(\mathrm{SF}_{6}\right)_{\mathrm{AVG}}$, are shown in Figs. 2 and 3, respectively. Also shown in each figure are $k_{660}(\mathrm{He})_{\mathrm{AVG}} \pm 2 \sigma_{\mathrm{He}}$ and $k_{660}\left(\mathrm{SF}_{6}\right)_{\mathrm{AVG}} \pm 2 \sigma_{\mathrm{SF} 6}$ at each wind speed, or the bounds showing range of $90 \%$ of the modelderived $k_{L}$ values (i.e., 900 out of the 1000 calculated transfer velocities at each $U$ lie within the dashed lines in each figure).

The fraction of the total observed variability that is explained by measurement errors can be estimated by calculating the standard errors in the experimental and simulated data sets. A least-squares linear regression of the $k_{L}$ values from the Monte Carlo simulations for each gas gives a standard error of the fit of 1.2 for $\mathrm{SF}_{6}$ and 1.1 for He. A least-squares linear regression of the experimental data for each gas gives standard errors of the fit of 2.3 and 1.9 for $\mathrm{SF}_{6}$ and $\mathrm{He}$, respectively. Taking the ratios of these values shows that the experimental measurement uncertainty explains $53 \%$ of the observed experimental variability for $\mathrm{SF}_{6}$ and $58 \%$ of the experimental variability for He. This indicates that half of the variability in the $k_{L}$ values is due to concentration measurement precision and not day-to-day changes in uncharacterized environmental conditions in the wind-wave tunnel.

In the experimental results and in the Monte Carlo simulations, the standard error for $k_{660}(\mathrm{He})$ is less than the standard error for $k_{660}\left(\mathrm{SF}_{6}\right)$. This result is counterintuitive given that the measurement uncertainty for $\mathrm{He}$ concentrations is more than double that for $\mathrm{SF}_{6}$. The reason for this difference reflects the interplay of Schmidt number, measurement time, and the logarithm term in Eq. (2). In the case where the total concentration change is small, the ratio term in Eq. (2) will 
be close to unity, making the logarithm small which amplifies the effect of the measurement error. Because $\mathrm{Sc}\left(\mathrm{SF}_{6}\right)$ is larger than $\mathrm{Sc}(\mathrm{He})$ by a factor of 8 and the transfer velocity scales as $\mathrm{Sc}^{-1 / 2}$, the concentration of $\mathrm{SF}_{6}$ in the wind-wave tunnel decreases a little under half as fast as He. This means for an equivalent experimental time period, the effect of errors in concentration will have a larger effect on $\mathrm{SF}_{6}$ than on He. The obvious solution to this problem is to run experiments over many hours so that both concentrations approach their equilibrium values. However, in situations where a set of gas transfer experiments must be conducted over a time span of a few days in a particular facility, running experiments for periods longer than a few hours can cause logistical complications.

The modeled concentrations can also be used to study the variability in values of $n$ deduced from measurements of $k_{L}$. Figure 4 shows $n$ calculated using Eq. (5) and $k_{L}\left(\mathrm{SF}_{6}\right)$ and $k_{L}(\mathrm{He})$ measured in the wind-wave tunnel plotted vesus $U$. Also shown in Fig. 4 is the average Schmidt number exponent, $n_{\mathrm{AVG}}$, calculated using the gas transfer velocities calculated from the Monte Carlo simulation above and Eq. (5). As expected for a clean surface, $n_{\mathrm{AVG}}=1 / 2$. Of more interest is the range in $n_{\mathrm{AVG}}$, here expressed as $n_{\mathrm{AVG}} \pm 2 \sigma_{n}$ where $\sigma_{n}$ is the standard deviation of the $1000 n$ values from the Monte Carlo simulation. The range in $n_{\mathrm{AVG}} \pm 2 \sigma_{n}$ spans the variability in $n$ calculated from the wind-tunnel data. This suggests that the variability observed in Fig. 4 is not due to environmental variability and merely reflects the sensitivity of $n$ to the experimental uncertainty in calculating $k_{L}$.

In the above analysis of the variability in $n$, it was assumed that $n$ was equal to $1 / 2$ and constant as a function of wind speed. However, laboratory data exists suggesting that $n$ is a function of $U$, decreasing from $2 / 3$ to $1 / 2$ as $U$ increases (Jähne et al., 1984; Zappa et al., 2004). Since understanding the dependence of $k_{L}$ on $n$ is important in determining a correct conceptual model for air-water gas transfer (Atmane et al., 2004) and in analyzing results from oceanic dual-tracer gas exchange experiments, it is often the case that gas transfer measurements are used to determine the dependence of $n$ on $U$.

The Monte Carlo method described above was used to assess how measurement errors might affect determining the functionality of $n$ with respect to $U$. First, it was assumed that $n$ was given by

$$
\begin{array}{ll}
n=0.67: & U<2 \mathrm{~ms}^{-1} \\
n=0.5+0.17 \exp \left(-\frac{U-2}{2}\right): & U \geq 2 \mathrm{~ms}^{-1}
\end{array}
$$

and then $k_{L}$ values were calculated for the gases methane $\left(\mathrm{CH}_{4}\right), \mathrm{SF}_{6}$, and He using Eq. (6) using $n$ calculated using Eq. (8) in place of the exponent $1 / 2$. Sc values for $\mathrm{CH}_{4}$ were taken from Wanninkhof (1992). Transfer velocities were calculated at $U=3 \mathrm{~m} \mathrm{~s}^{-1}, 4 \mathrm{~m} \mathrm{~s}^{-1}, 6 \mathrm{~m} \mathrm{~s}^{-1}, 8 \mathrm{~m} \mathrm{~s}^{-1}, 10 \mathrm{~m} \mathrm{~s}^{-1}$, and $12 \mathrm{~m} \mathrm{~s}^{-1}$. As before, 1000 concentration time series that included a $\pm 3 \%$ random error and had time steps as given

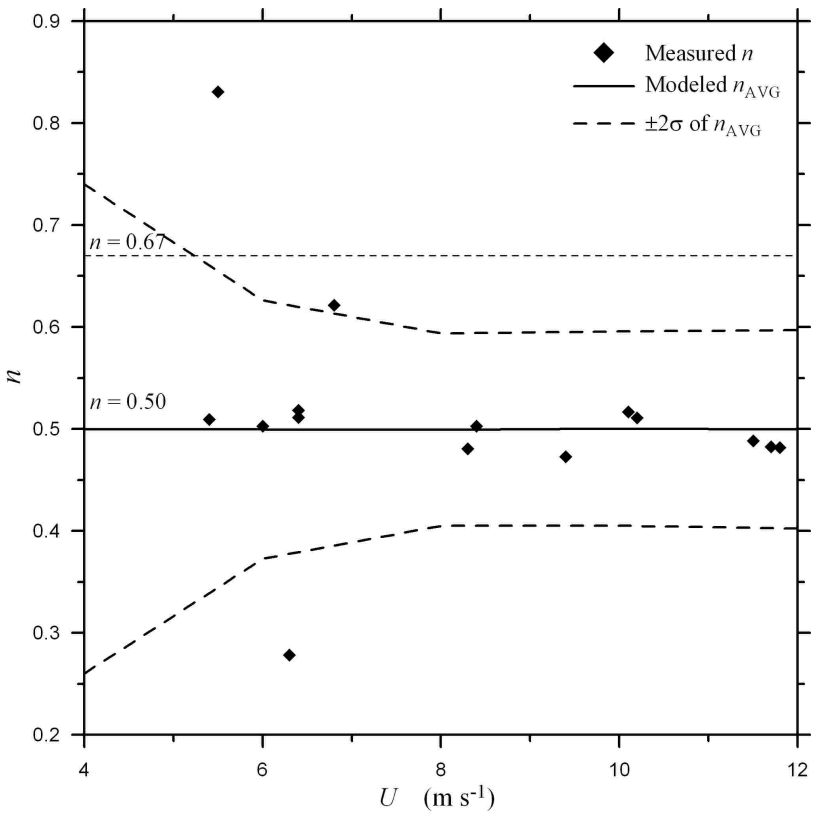

Fig. 4. The Schmidt number exponent, $n$, calculated using Eq. (5) and gas transfer velocity data for the gas pair $\mathrm{SF}_{6}$ and He measured in the Wallops Flight Facility wind/wave tunnel. Also shown in the figure is the average $n, n_{\mathrm{AVG}}$, and range of $n$ calculated using the Monte Carlo method and 1000 trial runs. The data key is shown on the figure where $\sigma$ is the standard deviation of the modeled $n$ values.

above in each value were generated for each gas. Then, $k_{L}$ was calculated using each concentration time series. An estimate of $n$ could then be derived using the gas pairs $\mathrm{CH}_{4} / \mathrm{SF}_{6}$, $\mathrm{CH}_{4} / \mathrm{He}$, and $\mathrm{SF}_{6} / \mathrm{He}$. Figure 5 shows $n_{\mathrm{AVG}}$ and $n_{\mathrm{AVG}} \pm 2 \sigma_{n}$ for all three gas pairs.

The choice of functional form for Eq. (8) was selected because it approximates the measured dependence of $n$ as a function of $U$ (Jähne et al., 1984; Zappa et al., 2004). Theoretical justification for assuming $n$ will decrease as $U$ increases can be made through heuristic appeals to eddy structure models for air-water gas transfer (Harriott, 1962). In these models, as the penetration depths and timescales of turbulence eddies at the water surface decrease, $n$ decreases from a maximum of 1 to a lower limit of $1 / 2$ - see Fig. 3 of Harriott (1962). Because it is known that penetration depth and timescales decrease with increasing levels of turbulence (Asher and Pankow, 1991a, b) and it is known that turbulence increases with increasing wind speed (Siddiqui et al., 2004), it is reasonable to assume that $n$ will decrease monotonically to the lower limit of $1 / 2$ as $U$ increases.

The top panel in Fig. 5 shows the results for $\mathrm{CH}_{4} / \mathrm{SF}_{6}$. Because the change in $\mathrm{Sc}$ value for these two gases is relatively small (e.g., $\mathrm{Sc}\left(\mathrm{CH}_{4}\right)=616 @ 293.15 \mathrm{~K}, \mathrm{Sc}\left(\mathrm{SF}_{6}\right)=948 @$ $293.15 \mathrm{~K}$ ), the calculation of $n$ is very sensitive to measurement error so the $\pm 2 \sigma_{n}$ range is large and if only a few experiments were conducted, it is doubtful the functionality of 


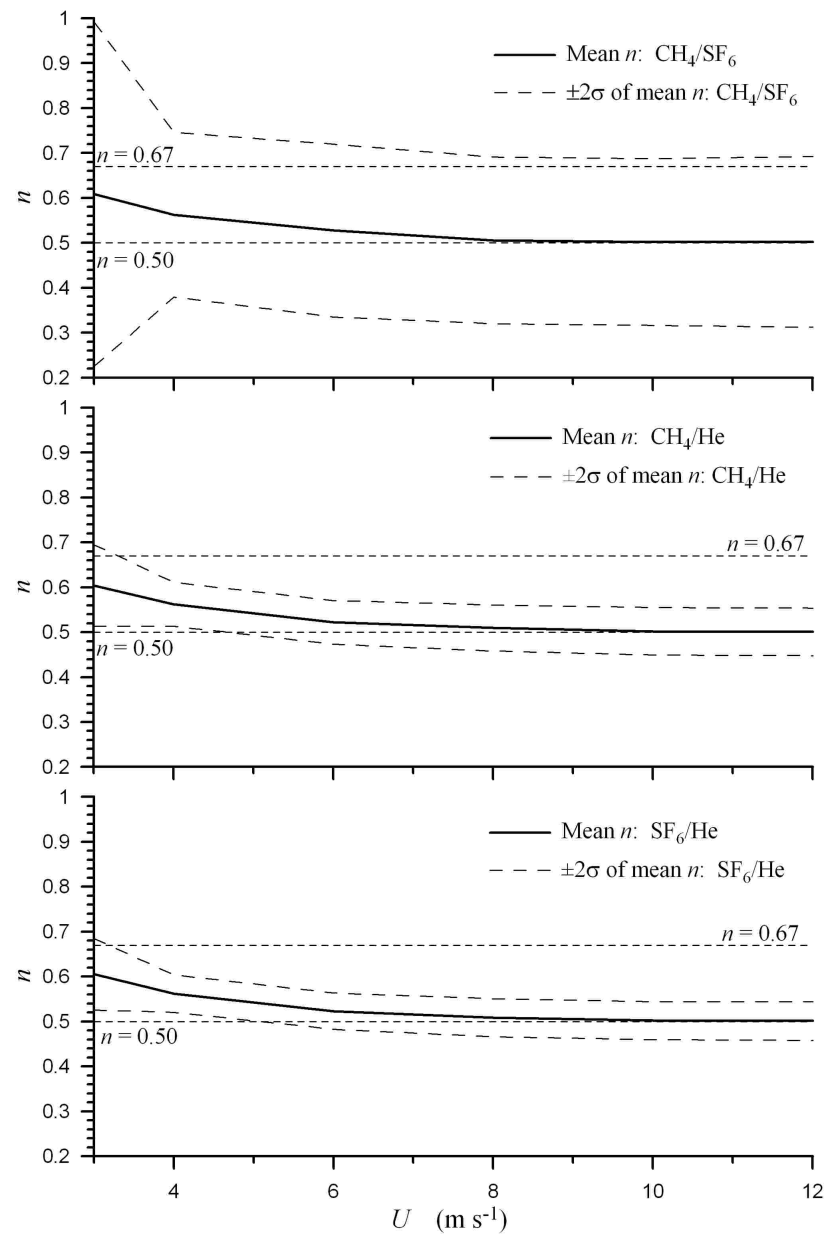

Fig. 5. Plots of the expected experimental variance in Sc exponent $n$ calculated using Eq. (5) and transfer velocities from the Monte Carlo procedure described in the text plotted as a function of wind speed, $U$, for the gas pairs: methane $\left(\mathrm{CH}_{4}\right)$ and sulfur hexafluoride $\left(\mathrm{SF}_{6}\right)$, top panel; $\mathrm{CH}_{4}$ and helium $(\mathrm{He})$, middle panel; and $\mathrm{He}$ and $\mathrm{SF}_{6}$, bottom panel. The model assumes the uncertainties in gas concentrations are uncorrelated. The data key for each is shown on each figure.

$n$ given by Eq. (8) would result. The situation improves for both gas pairs involving $\mathrm{He}$, mainly because $\mathrm{Sc}(\mathrm{He})=149$ at $293.15 \mathrm{~K}$. However, the variability in the calculated $n$ values is still large and accurately resolving the dependence of $n$ on wind speed would require a significant number of experiments be conducted.

\section{Oceanic purposeful dual-tracer measurements}

The Monte Carlo method described in the previous section can also be applied to the purposeful dual-tracer method PDTM. However, in the case of PDTM data analysis, in addition to the measurement uncertainties in the gas concentrations, the uncertainty in the mixed layer depth, $h$, must also be taken into account. As discussed by Wanninkhof et al. (2004), the analysis of PDTM data proceeds by analyzing discrete segments of a times series of concentrations for the two gases over intervals where the wind speed was relatively constant. Although nonsteady wind speeds are recognized as having an effect on PDTM analysis (Wanninkhof et al., 2004), including variable wind speeds is beyond the scope of this paper and this study will assume steady wind speeds.

For the simulations performed here, it was assumed that the concentrations of both $\mathrm{SF}_{6}$ and ${ }^{3} \mathrm{He}$ could be measured in the ocean with a precision of $\pm 2 \%$ (R. Wanninkhof, NOAA Atlantic Oceanographic and Meteorological Laboratory, Miami Florida, personal communication). Defining the uncertainty in $h$ is more problematic, especially considering that there have been two distinct types of oceanic PDTM experiments conducted. PDTM experiments have been conducted in well-mixed shallow-water coastal environments, where the mixed-layer depth is defined by the water depth (e.g., Wanninkhof et al., 1993; Watson et al., 1991). In these cases, where depth is typically measured using an ADCP, the uncertainty in $h$ is due to tides and averaging over the depth bin in the ADCP. Therefore, the resulting uncertainties in $h$ are on order of $\pm 10 \%$. More recently, PDTM experiments have been conducted in the open ocean, where $h$ is defined as the depth of the mixed-layer (Ho et al., 2006; Nightingale et al., 2000a; Wanninkhof et al., 1997, 2004). In these cases the uncertainty in $h$ was assumed to be $\pm 20 \%$ based on the uncertainty in extracting the mixed-layer depth from CTD profiles (D. Ho, University of Hawaii, personal communication).

Unfortunately, the precision in gas concentration analysis for the oceanic dual-tracer data is different than the precision in concentration analysis for the wind-tunnel data discussed above. This makes direct comparison of the data problematic, but because of differences in instrumentation and analytical techniques, it is not correct to use the same experimental precisions for each set of experiments.

The Monte Carlo simulations were carried out by calculating the transfer velocities of $\mathrm{SF}_{6}$ and $\mathrm{He}, k_{L}\left(\mathrm{SF}_{6}\right)$ and $k_{L}(\mathrm{He})$, respectively, at for $U=4 \mathrm{~m} \mathrm{~s}^{-1}, 8 \mathrm{~m} \mathrm{~s}^{-1}, 12 \mathrm{~m} \mathrm{~s}^{-1}$ and $16 \mathrm{~m} \mathrm{~s}^{-1}$ using the $U^{2}$ power law dependence proposed by Wanninkhof (1992). Assuming that $h=50 \mathrm{~m}$ (and that $A / V=1 / h$ ) allowed Eq. (7) to be used with these $k_{L}$ values to generate concentrations for $\mathrm{SF}_{6}$ and ${ }^{3} \mathrm{He}$ at discrete times. Concentration pairs were calculated at $t=0$ and at times with $\Delta t$ in the range of 1-2 days that included the independent measurement uncertainties. Then, an experimental $h$ was chosen that included the effect of uncertainty. The $h$ and concentrations were used in Eq. (3) to calculate $k_{L}\left({ }^{3} \mathrm{He}\right)$. As was done previously, 1000 sets of concentrations were generated at each $U$ and a mean transfer velocity normalized to $\mathrm{Sc}=660, k_{660}\left({ }^{3} \mathrm{He}\right)_{\mathrm{AVG}}$, and associated standard deviation, $\sigma_{3 \mathrm{He}}$, were calculated. One set of $k_{660}\left({ }^{3} \mathrm{He}\right)$ values were calculated using an uncertainty of $\pm 10 \%$ for $h$ to simulate the measurement variability in the shallow-water coastal PDTM 


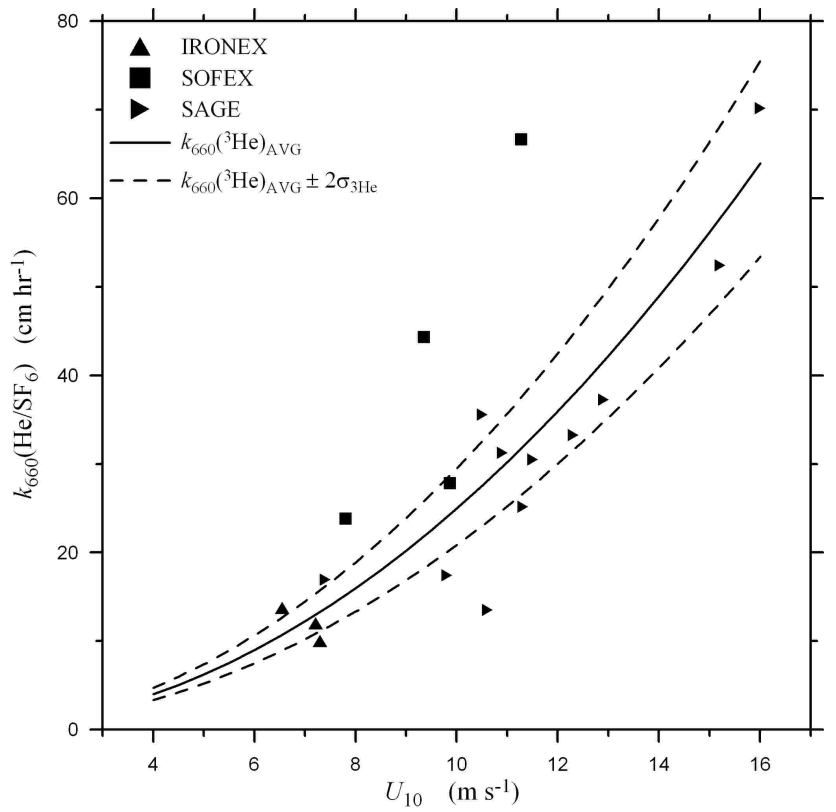

Fig. 6a. Air-sea gas transfer velocities of helium-3 normalized to $\mathrm{Sc}=660, k_{660}\left({ }^{3} \mathrm{He}\right)$, measured using the purposeful dual-tracer method in deep-water open ocean environments. Also shown are $k_{660}\left({ }^{3} \mathrm{He}\right)$ values produced by the Monte Carlo simulations described in Sect. 4. The data key is shown on the figure. Refer to the caption of Fig. 1 for explanation of experimental codes.

experiments and a separate set of $k_{660}\left({ }^{3} \mathrm{He}\right)$ values were calculated using an uncertainty in $h$ of $\pm 20 \%$ to simulate the open ocean PTDM experiments. Figure 6 shows the data from Fig. 1 separated in the coastal and open-ocean PTDM experiments along with the $k_{660}\left({ }^{3} \mathrm{He}\right)_{\mathrm{AVG}}$, and the range of the model results given as $k_{660}(\mathrm{He})_{\mathrm{AVG}} \pm 2 \sigma_{3 \mathrm{He}}$ calculated using the relevant uncertainties in the gas concentrations and $h$.

Following the data analysis for the wind-wave tunnel data in the previous section, a least-squares regression of all the coastal PDTM field measurements in Fig. 6a (i.e., the North Sea, Georges Bank, FSLE, and ASGAMAGE data sets) of $k_{660}\left({ }^{3} \mathrm{He}\right)$ versus $U^{2}$ gives a standard error of the fit of 7.0. A least-squares regression of all the $k_{660}\left({ }^{3} \mathrm{He}\right)$ values from the Monte Carlo simulation with a $10 \%$ uncertainty in $h$ gives a standard error of the fit of 3.0. Therefore, these results indicate that $43 \%$ of the observed variability in the coastal PDTM results is due to uncertainty in gas concentrations and $h$. In the case of the open-ocean deep-water PDTM experiments in Fig. $6 \mathrm{~b}$ (i.e., the IRONEX, SAGE, and SOFEX data sets), the standard error of the fit of the experimental data is 11.2. The regression of the Monte Carlo simulations run with a $20 \%$ uncertainty in $h$ gives a standard error of 4.9, which indicates that $44 \%$ of the observed variability is explained by the measurement uncertainties in the concentrations and $h$ (interestingly, if the SOFEX data set is excluded from this analysis, the experimental standard error for the PDTM data decreases to 5.9 and the fraction of the error explained by

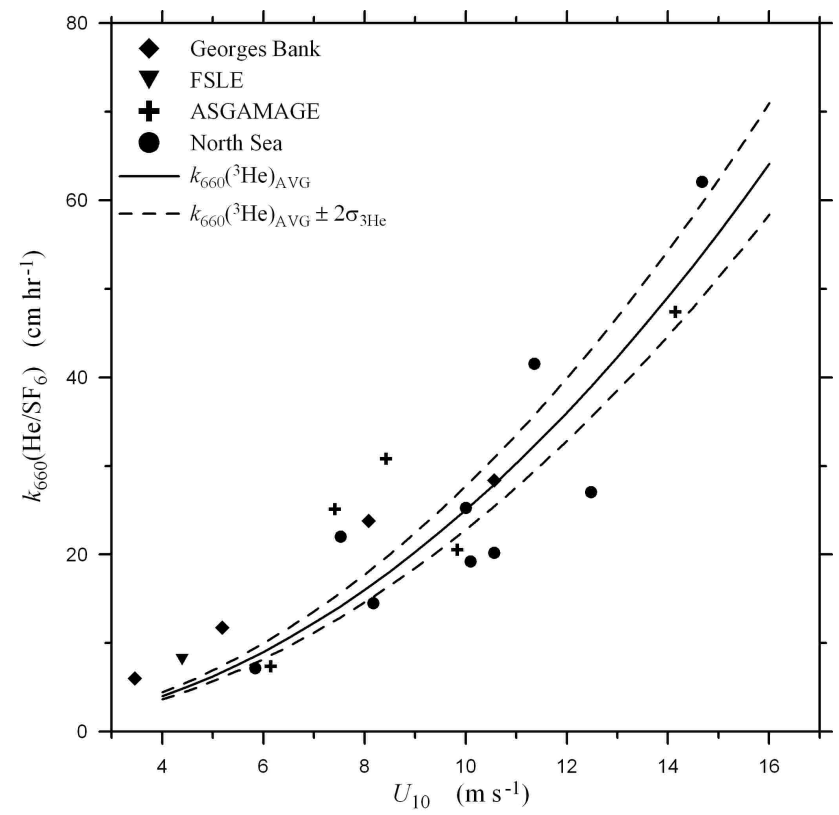

Fig. 6b. Air-sea gas transfer velocities of helium-3 normalized to $\mathrm{Sc}=660, k_{660}\left({ }^{3} \mathrm{He}\right)$, measured using the purposeful dual-tracer method in shallow-water coastal environments. Also shown are $k_{660}\left({ }^{3} \mathrm{He}\right)$ values produced by the Monte Carlo simulations described in Sect. 4. The data key is shown on the figure. Refer to the caption of Fig. 1 for explanation of experimental codes.

the measurement errors rises to $83 \%$ ). This suggests that environmental variability may not play as large a role as supposed, and that measurement uncertainty may account for approximately half of the observed scatter in the field measurements. Given the large difference between the precision of the gas concentration measurements and $h$, it is was found that uncertainty in $h$ has the largest effect on $k_{L}\left({ }^{3} \mathrm{He}\right)$. In particular, $h$ accounts for $72 \%$ of the standard error in the modeled transfer velocities for the open-ocean deep-water runs and $57 \%$ of the standard error for the coastal runs.

\section{Conclusions}

The simple Monte Carlo model used here does not account for all sources of experimental variability in measuring airwater gas transfer velocities. However, it is instructive that using realistic values for the uncertainties in the concentration measurements accounts for over half of the observed variability in transfer velocities measured in wind-wave tunnels. Using the uncertainties in gas concentrations and mixed-layer depth in PDTM experiments also results in explaining approximately half of the observed variability of the field measurements. This suggests that in both the field and laboratory there is less variability in the forcing mechanisms driving gas exchange than would be assumed on inspection of the data. This conclusion implies that the overall pattern 
of the data in Fig. 1 is close to the "true" functional dependence of $k_{L}$ on wind speed. In turn, this is relatively good news for those who hope to develop a robust method for parameterizing gas transfer in terms of an easily measured environmental variable. However, it also points out the difficulty associated with making the measurements used to validate these parameterizations, especially in regards to determining the functional dependence of $k_{L}$ on Schmidt number.

Specific recommendations for reduction of variability in laboratory experiments include reducing measurement errors in gas concentrations, since these errors can account for half of the observed variability in the transfer velocities. This is especially true when conducting experiments designed to determine $n$. In cases where increasing the precision of the concentration measurements is not possible, increasing the length of time over which concentration decreases are measured reduces the effect of uncertainty in concentrations.

In the case of PDTM experiments conducted in stratified waters, where the mixed layer depth must be determined by CTD cast or other method, these results show that transfer velocities are particularly sensitive to uncertainty in $h$. In these cases reducing the uncertainty in concentrations would have little effect on the experimental noise and particular attention should be paid to measuring the mixed-layer depth. The results from PDTM experiments conducted in well-mixed shallow waters show a higher fraction of the observed experimental variability that is not accounted for by the measurement uncertainties. This suggests that the effects of variability in the forcing mechanisms are higher in these situations, and particular emphasis should be placed on measuring the forcing functions for gas exchange such as wind stress and wave breaking.

Acknowledgements. This work was funded by the National Science Foundation under grant OCE-0425305. The comments of the referees were very helpful in preparation of this paper and I thank them for their input.

Edited by: J. Kaiser

\section{References}

Asher, W. E. and Litchendorf, T. M.: Visualizing near-surface CO2 concentration fluctuations using laser-induced fluorescence, Exp. in Fluids, doi:10.1007/s00348-008-0554-9, in press, 2009.

Asher, W. E. and Pankow, J. F.: The effect of surface films on concentration fluctuations close to a gas/liquid interface, in: AirWater Mass Transfer, edited by: Wilhelms, S. E., and Gulliver, J. S., American Society of Civil Engineering, New York, 68-80, 1991a.

Asher, W. E. and Pankow, J. F.: Prediction of gas/water mass transport coefficients by a surface renewal model, Env. Sci. Tech., 25, 1294-1300, 1991b.

Atmane, M. A., Asher, W. E., and Jessup, A. T.: On the use of the active infrared technique to infer heat and gas transfer veloc- ities at the air-water free surface, J. Geophys. Res.-Oceans, 109 (C08S14), doi:10.1029/2003JC001805, 2004.

Davies, J. T.: Turbulence Phenomena, Academic Press, New York, 412 pp., 1972.

Frew, N. M., Goldman, J. C., Dennett, M. R., and Johnson, A. S.: Impact of phytoplankton-generated surfactants on air-sea gas exchange, J. Geophys. Res.-Oceans 95C, 3337-3352, 1990.

Harriott, P.: A random eddy modification of the penetration theory, Chem. Eng. Sci. 17, 149-154, 1962.

Herlina and Jirka, G. H.: Application of LIF to investigate gas transfer near the air-water interface in a grid-stirred tank, Exp. Fluids, 37(3), 341-349, 2004.

Herlina and Jirka, G. H.: Experiments on gas transfer at the airwater interface induced by oscillating grid turbulence, J. Fluid Mech. 594, 183-208, 2008.

Ho, D. T., Law, C. S., Smith, M. J., Schlosser, P., Harvey, M., and Hill, P.: Measurements of air-sea gas exchange at high wind speeds in the Southern Ocean: Implications for global parameterizations, Geophys. Res. Lett., 33 L16611, doi:10.1029/2006GL026817, 2006.

Jacobs, C., Kjeld, J. F., Nightingale, P., Upstill-Goddard, R., Larsen, S., and Oost, W.: Possible errors in $\mathrm{CO} 2$ air-sea transfer velocity from deliberate tracer releases and eddy covariance measurements due to near-surface concentration gradients, J. Geophys. Res.-Oceans 107 (L3128), doi:10.1029/2001JC000983, 2002.

Jacobs, C. M. J., Kohsiek, W., and Oost, W. A.: Air-sea fluxes and transfer velocity of $\mathrm{CO} 2$ over the North Sea: results from ASGAMAGE, Tellus B, 51(3), 629-641, 1999.

Jähne, B., Heinz, G., and Dietrich, W.: Measurement of the diffusion coefficients of sparingly soluble gases in water, J. Geophys. Res.-Oceans 92, 10767-10 776, 1987.

Jähne, B., Huber, W., Dutzi, A., Wais, T., and Ilmberger, J.: Wind/wave tunnel experiments on the Schmidt number and wave field dependence of air-water gas exchange, in: Gas Transfer at Water Surfaces, edited by: Brutsaert, W. and Jirka, G. H., Reidel, Dordrecht, 303-310, 1984.

King, D. B. and Saltzman, E. S.: Measurement of the diffusion coefficient of sulfur hexafluoride in water, J. Geophys. Res.-Oceans, 100, 7083-7088, 1995.

McGillis, W. R., Edson, J. B., Hare, J. E., and Fairall, C. W.: Direct covariance air-sea $\mathrm{CO} 2$ fluxes, J. Geophys. Res.-Oceans, 106(C8), 16 729-16745, 2001a.

McGillis, W. R., Edson, J. B., Ware, J. D., Dacey, J. W. H., Hare, J. E., Fairall, C. W., and Wanninkhof, R. Carbon dioxide flux techniques performed during GasEx-98, Mar. Chem., 75, 267280, 2001b.

McGillis, W. R., Edson, J. B., Zappa, C. J., Ware, J. D., McKenna, S. P., Terray, E. A., Hare, J. E., Fairall, C. W., Drennan, W., Donelan, M., DeGrandpre, M. D., Wanninkhof, R., and Feely, R. A.: Air-sea CO2 exchange in the equatorial Pacific, J. Geophys. Res.-Oceans, 109, 2004.

McKenna, S. P. and McGillis, W. R.: The role of free-surface turbulence and surfactants in air-water gas transfer, Int. J. Heat Mass Trans., 47(3), 539-553, 2004.

Münsterer, T. and Jähne, B.: LIF measurements of concentration profiles in the aqueous mass boundary layer, Exp. Fluids, 25 190-196, 1998.

Nightingale, P. D., Liss, P. S., and Schlosser, P.: Measurements of air-sea gas transfer during an open ocean algal bloom, Geophys. 
Res. Lett., 27(14), 2117-2120, 2000a.

Nightingale, P. D., Malin, G., Law, C. S., Watson, A. J., Liss, P. S., Liddicoat, M. I., Boutin, J., and Upstill-Goddard, R. C.: In situ evaluation of air-sea gas exchange parameterizations using novel conservative and volatile tracers, Global Biogeochem. Cy., 14, 373-387, 2000b.

Siddiqui, M. H. K., Loewen, M. R., Asher, W. E., and Jessup, A. T.: Coherent structures beneath wind waves and their influence on air-water gas transfer, J. Geophys. Res.-Oceans 109, C03024, doi:03010.01029/02002JC001559, 2004.

Wanninkhof, R.: Relationship between wind speed and gas exchange over the ocean, J. Geophys. Res.-Oceans, 97, 7373-7382, 1992.

Wanninkhof, R., Asher, W. E., Weppernig, R., Chen, H., Schlosser, P., Langdon, C., and Sambrotto, R.: Gas transfer experiment on Georges Bank using two volatile deliberate tracers, J. Geophys. Res.-Oceans, 98, 20 237-20 248, 1993.
Wanninkhof, R., Hitchcock, G., Wiseman, W., Ortner, P., Asher, W., Ho, D., Schlosser, P., Dickson, M.-L., Anderson, M., Masserini, R., Fanning, K., and Zhang, J.-Z.: Gas exchange, dispersion, and biological productivity on the west Florida shelf: Results from a Lagrangian tracer study, Geophys. Res. Lett., 24, 1767-1770, 1997.

Wanninkhof, R., Sullivan, K. F., and Top, Z. Air-sea gas transfer in the Southern Ocean, J. Geophys. Res.-Oceans, 109, C08S19, doi:10.1029/2003JC001767, 2004.

Woodrow Jr., P. T., and Duke, S. R.: Laser-induced fluorescence studies of oxygen transfer across unsheared flat and wavy airwater interfaces, Ind. Eng. Chem. Res., 40, 1985-1995, 2001.

Zappa, C. J., Asher, W. E., Jessup, A. T., Klinke, J., and Long, S. R.: Microbreaking and the enhancement of air-water gas transfer velocities, J. Geophys. Res.-Oceans, 109, C08S16, doi:10.1029/2003JC001897, 2004. 